\title{
Vilazodone-Tacrine Hybrids as Potential Anti-Alzheimer Agents: QSAR, Molecular Docking, and Molecular Dynamic (MD) Simulation Studies
}

\author{
Hanieh Abbasi ${ }^{1}$, Masood Fereidoonnezhad ${ }^{1,2, *}$ (D) Zohreh Mirveis $^{1}$ \\ 1 Department of Medicinal Chemistry, School of Pharmacy, Ahvaz Jundishapur University of Medical Sciences, Ahvaz, \\ Iran; hanieabasi6@gmail.com (H.A.), shohre001@gmail.com (Z.M.); \\ 2 Toxicology Research Center, Medical Basic Sciences Research Institute, Ahvaz Jundishapur University of Medical \\ Sciences, Ahvaz, Iran; Fereidoonnezhad-m@ajums.ac.ir (M.F.); \\ * Correspondence: Fereidoonnezhad-m@ajums.ac.ir;
}

Scopus Author ID 35490192400

Received: 1.03.2021; Revised: 3.04.2021; Accepted: 7.04.2021; Published: 20.04.2021

\begin{abstract}
Alzheimer's disease (AD) is a type of functional impairment of the brain, which gently degrades the patients' mental potency. The goal pursued in this study is to introduce novel drug candidates as acetylcholinesterase (hAChEIs) and butyl cholinesterase (hBuChE) inhibitors in the treatment of $\mathrm{AD}$. In this study, more than 100 Vilazodone-Tacrin hybrids have been designed to inhibit $\mathrm{hAchE}$ and $\mathrm{hBuChE}$. A series of chemical methods such as multiple linear regression (MLR) and GAPLS were used to create relationships between structural features in these compounds and their hAchE/ hBuChE inhibitory activity. Molecular docking studies against hAChE (PDB ID: 1ACJ) and hBuChE (PDB ID: 4BDS) indicates the important amino acids inside the active site cavity that are responsible of essential interactions were Gly441, Ser122, Trp84, Asp72, Tyr130, and Gly117 for hAChE and Glu197, Thr120, Gly121, Asn83, Tyr332, Trp430, Trp82 and Asp70 for hBuChE. The best regression equation was obtained from GA-PLS, which predicts $92 \%$ of variances. Molecular dynamic (MD) simulations were also studied. The reliability, accuracy, and predictability of the proposed QSAR models were evaluated. Finally, an in silico screening study was conducted, and some compounds were introduced as logical candidates as novel anti-Alzheimer agents.
\end{abstract}

Keywords: anti-Alzheimer; cholinesterase ( $h$ AchE and $h$ BuchE) inhibitors; molecular docking; QSAR; Molecular Dynamic (MD) simulation.

(C) 2021 by the authors. This article is an open-access article distributed under the terms and conditions of the Creative Commons Attribution (CC BY) license (https://creativecommons.org/licenses/by/4.0/).

\section{Introduction}

Alzheimer's Disease (AD) is a brain disease that gradually degrades the patients' mental abilities [1]. According to published reports, the number of people affected by AD is expected to increase to 63.37 million in 2030 [2]. With the advancement of the disease, brain neurons are more likely to be harmed, which results in the destruction of nerve cells in the parts involved in thinking, learning, and memory. Other changes that occur after losing a nerve cell are inflammation and atrophy [1,2].

The cause of $\mathrm{AD}$ is based on three hypotheses, including cholinesterase enzymes, betaamyloid $(\beta A)$ protein, and chronic inflammatory theory. Several studies have shown that amyloid plaques may be the only contributing factor to AD disease [3,4]. Based on existing assumptions, various types of drugs, including $A \beta$ accumulation inhibitors, anti-Tau drugs, and anti-inflammatory and cholinergic enhancers, are available. 
Since the reduction of cholinergic neuronal activity is an apparent feature of AD, cholinesterase drugs greatly treat this disease [5]. Currently, acetylcholinesterase inhibitors (AChEI) and butyrylcholinesterase (BuChEI) inhibitors are known as the primary treatment line [6]. By inhibiting acetylcholinesterase and butyrylcholinesterase, the tacrine increases the rate of acetylcholine $(\mathrm{ACh})$ and butyrylcholine $(\mathrm{BuCh})$ availability in which the cholinergic neurotransmitter increases. However, its high side effects and toxicity cannot be extensively used in the treatment [3]. Studies have shown that by synthesizing the tacrine derivatives, its safety can increase and can be used as a scaffolding to expand anti-Alzheimer factors [6].

Today, due to time-consuming and expensive experimental study methods, drug design techniques are considered by computer software as one of the most important and useful tools for rational drug development $[7,8]$.

In this paper, molecular modeling techniques including MD simulation, molecular docking, and QSAR were used to institute a meaningful relationship between VilazodoneTacrine hybrids structures and their inhibitory activity on $h \mathrm{AChE}$ and $h \mathrm{BuChE}$ targets. To achieve the exact molecular connection models, molecular modeling simulation techniques were performed on all the data set components and designed compounds. Finally, using in silico screening study, some novel compounds were introduced as logical candidates as antiAlzheimer agents.

\section{Materials and Methods}

\subsection{Data set.}

The data used in this study included 39 derivatives of the Vilazodone-Tacrine hybrids as choline esterase inhibitors [3]. The biological activity and structural characteristics of these compounds are listed in Table 1. The data are transformed into a logarithmic scale of the pIC 50 , which is ultimately used for QSAR studies.

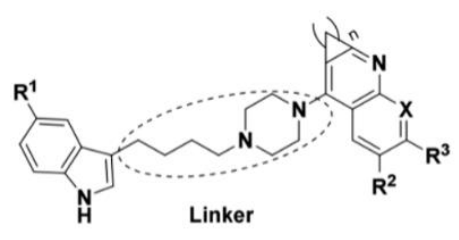

(A1-A23)

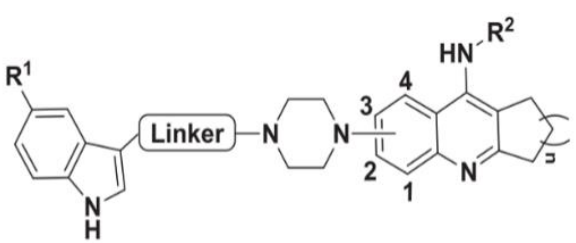

(B1-B16)

Figure 1. Chemical structure of the compounds used in this study. R1, R2, R3, X, n are shown in Table 1.

Table 1. The cross-validated predicted and experimental activity of compounds, as well as docking binding energy on $h \mathrm{AchE}$ and $h \mathrm{BuchE}$ targets.

\begin{tabular}{|c|c|c|c|c|c|c|c|c|c|c|c|c|}
\hline \multirow[b]{2}{*}{ Name } & \multirow[b]{2}{*}{ Linker } & \multirow[b]{2}{*}{$\mathbf{R}^{1}$} & \multirow[b]{2}{*}{$\mathbf{R}^{2}$} & \multirow[b]{2}{*}{$\mathbf{R}^{3}$} & \multirow[b]{2}{*}{$\mathbf{X}$} & \multirow[b]{2}{*}{$\mathbf{n}$} & \multicolumn{3}{|c|}{$h$ AChE } & \multicolumn{3}{|c|}{$h \mathrm{BuChE}$} \\
\hline & & & & & & & $\begin{array}{l}\text { pIC } 50 \\
\text { pred }\end{array}$ & leverage & $\begin{array}{c}\Delta \mathbf{E} \\
\text { (kcal/ } \\
\text { mol) }\end{array}$ & $\begin{array}{c}\text { pIC } 50 \\
\text { pred }\end{array}$ & leverage & $\begin{array}{c}\Delta \mathbf{E} \\
\text { (kcal/ } \\
\text { mol) }\end{array}$ \\
\hline $\mathbf{A 1}$ & & $\mathrm{CN}$ & $\mathrm{H}$ & $\mathrm{H}$ & $\mathrm{H}$ & 4 & 5.757 & 0.124 & -14.38 & 5.200 & 0.052 & -12.55 \\
\hline A2 & & $\mathrm{CN}$ & $\mathrm{H}$ & $\mathrm{H}$ & $\mathrm{N}$ & 4 & 5.867 & 0.131 & -15.39 & 4.581 & 0.219 & -13.31 \\
\hline A3 & & $\mathrm{CN}$ & $\mathrm{H}$ & $\mathrm{H}$ & $\mathrm{H}$ & 3 & 5.597 & 0.152 & -15.3 & 5.376 & 0.029 & -11.07 \\
\hline A4 & & $\mathrm{CN}$ & $\mathrm{H}$ & $\mathrm{H}$ & $\mathrm{H}$ & 5 & 5.712 & 0.125 & -16.45 & 5.548 & 0.227 & -11.31 \\
\hline
\end{tabular}


https://doi.org/10.33263/BRIAC121.588607

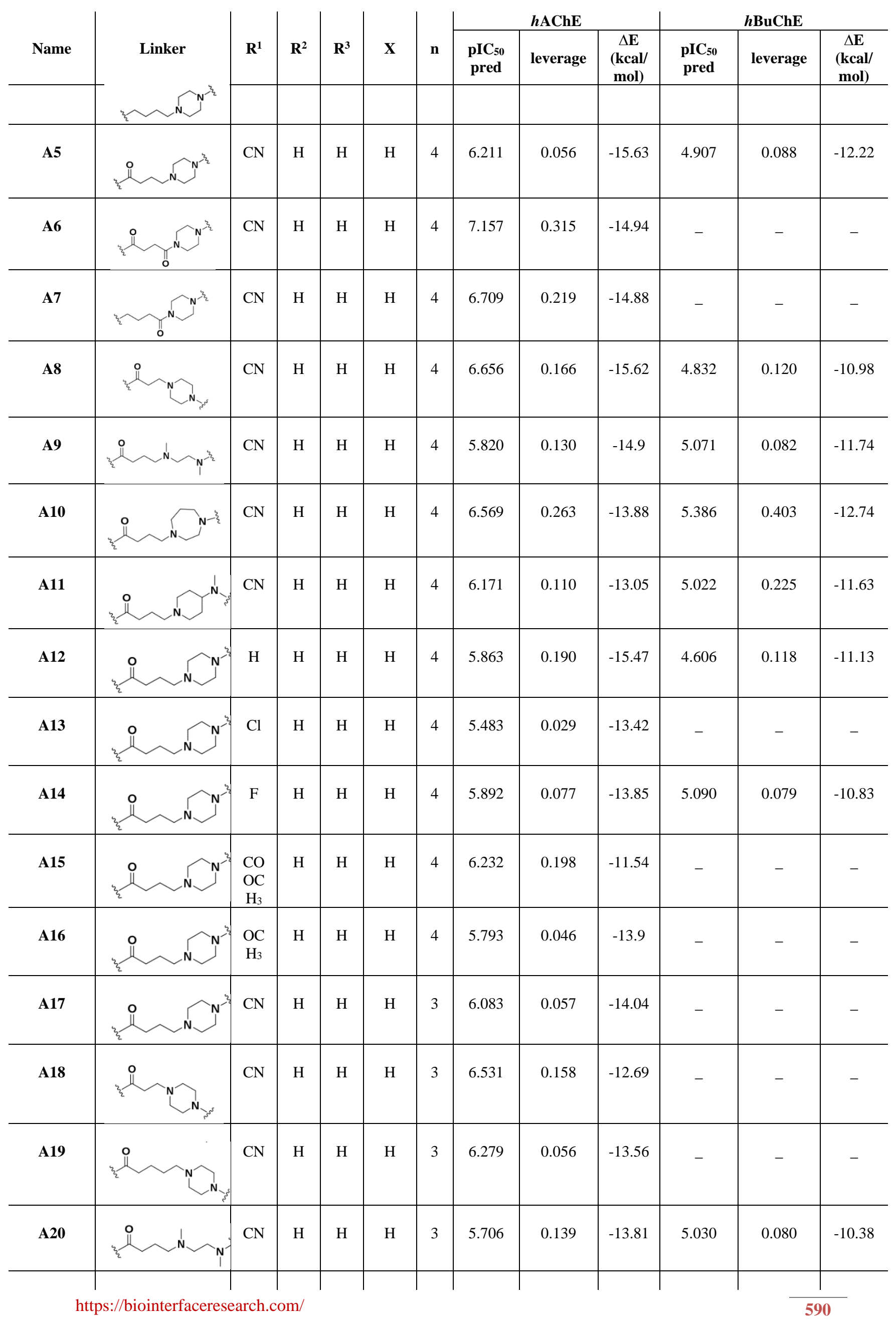


https://doi.org/10.33263/BRIAC121.588607

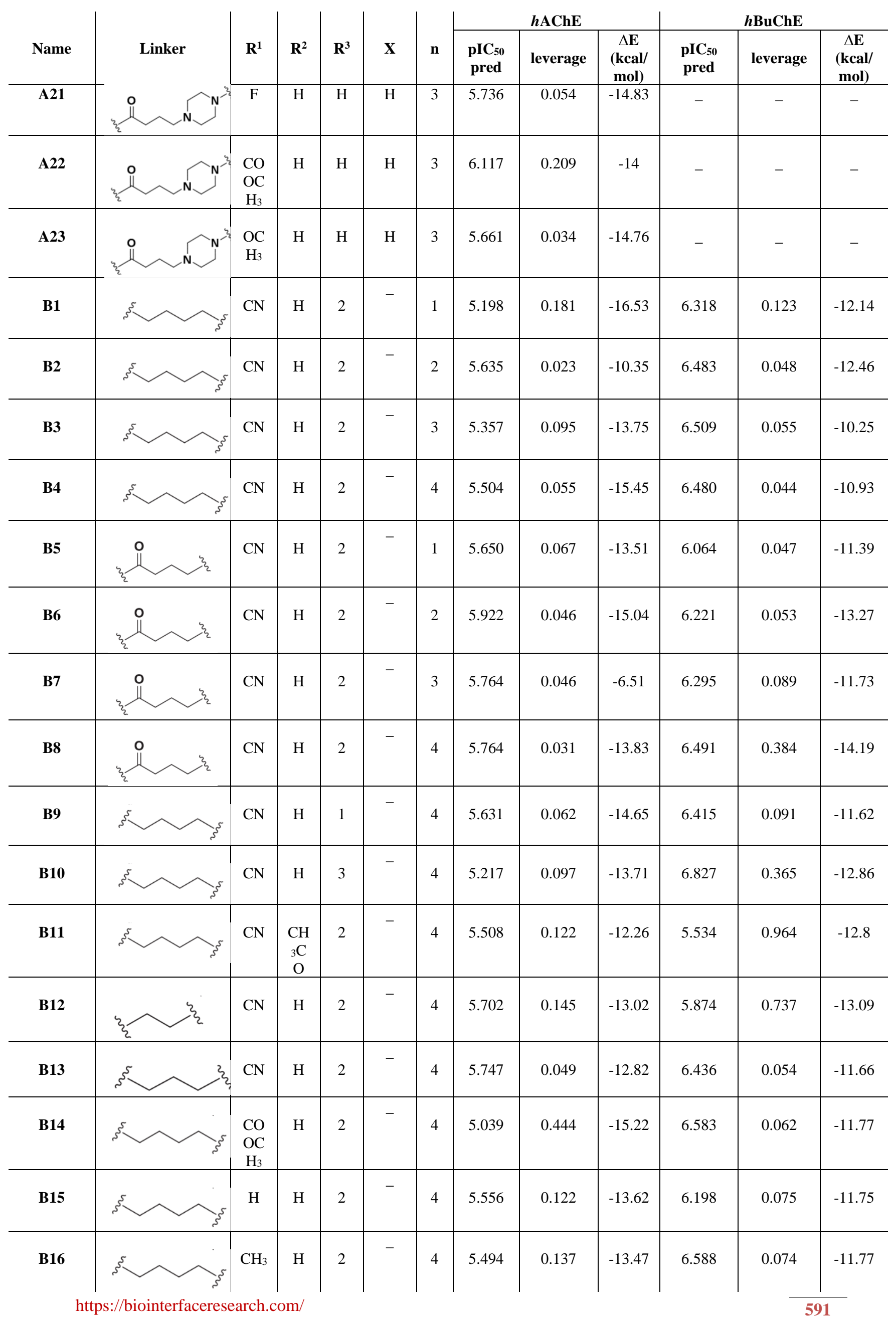




\subsection{Molecular descriptors.}

Two-dimensional structures of the ligands were created using ChemBioDraw 12.0 software [9]. The ligands were subjected to minimization procedures using an in-house TCL script using Hyperchem (Version 8, Hypercube Inc., Gainesville, FL, USA). Each ligand was optimized with different minimization methods such as molecular mechanics (MM+) followed by a quantumbased semi-empirical method (AM1) using the Hyperchem package. Z-matrices of the structures were processed by the software and transferred to the Gaussian 98 program [10]. An outsized number of molecular descriptors were computed using Gaussian 98, Hyperchem, and Dragon package [11]. Some chemical parameters containing molecular volume (V), hydration energy (HE), hydrophobicity $(\operatorname{LogP})$, molecular surface area $(\mathrm{SA})$, and molecular polarizability (MP) were calculated using Hyperchem Software. Highest occupied molecular orbital (HOMO) and lowest unoccupied molecular orbital (LUMO) energies, the foremost positive and also the most negative net atomic charges, the average absolute atomic charge, and molecular dipole moment were calculated by Gaussian98 software. Quantum chemical indices hardness $(\eta=0.5$ $(\mathrm{HOMO}+\mathrm{LUMO}))$; softness $(\mathrm{S}=1 / \eta)$; electronegativity $(\chi=-0.5(\mathrm{HOMO}-\mathrm{LUMO}))$; and electrophilicity $(\omega=\chi 2 / \eta)$ were calculated consistent with the equations proposed by Thanikaivelan et al. [12]. Dragon calculated different topological, geometrical, empirical, and constitutional descriptors for every molecule. 2D autocorrelations, atom-centered fragments, and functional groups were also calculated using the Dragon.

\subsection{Variable importance in the projection (VIP).}

Variable important in projection (VIP) was employed to survey the relative importance of the variable that appeared within the final model obtained by the GA-PLS method [13]. The importance of terms within the PLS model is reflected in VIP values. Because it was explained by Erikson et al., X-variables (predictor variables) may be classified under their relevance in explaining y (predicted variable), so that VIP $>1.0$ and VIP $<0.8$ mean highly or less influential, respectively, and $0.8<\mathrm{VIP}<1.0$ means moderately influential [14].

\subsection{Docking procedure.}

The docking studies were executed utilizing an in-house batch script (DOCKFACE) [15, 16] of Autodock 4.2. For the docking procedure, each ligand was optimized with $\mathrm{MM}+$, then the AM1 minimization method using HyperChem 8. The atoms' partial charges were calculated using the Gasteiger Marsili procedure implemented within the AutoDock Tools package [17]. Non-polar hydrogens of compounds were merged, and so rotatable bonds were assigned. The output structures were converted to PDBQT using MGLtools 1.5.6 [18].

The three-dimensional crystal structure of $h \mathrm{AChE}$ (PDB ID:1ACJ), and $h \mathrm{BuChE}$ (4BDS) were derived from protein data bank (http://www.rcsb.org/pdb/home/home.do) [19]. Water and co-crystal ligand molecules were eliminated from the structures, and missing hydrogens were added and after determining the Kollman united atom charges, non-polar hydrogens were elided into their corresponding carbons using Autodock Tools [20]. Then, solvation parameters were appointed to every protein atom. Among the three different search algorithms accomplished by Autodock 4.2, the commonly used Lamarckian Genetic Algorithm (LGA) was applied [21, 22]. The enzymes were converted into PDBQT using MGLTOOLS 1.5.6. For Lamarckian GA, 100 No. of GA run, 27000 maximum generations; the maximum number of 2,500,000 energy evaluations, 150 population size, the mutation rate of 0.02 ; and a crossover rate of 0.8 were applied. 
The grid maps of the receptors were calculated using AutoGrid tools of Autodock 4.2. The grid's dimensions were set in an exceedingly include the active site and portions of the encircling surface. A grid box of $50 \times 46 \times 46$ points in $\mathrm{x}, \mathrm{y}$, and $\mathrm{z}$-direction with a grid spacing of $0.375 \AA$ was built for all targets. The center of the grid box in $\mathrm{x}, \mathrm{y}$, and $\mathrm{z}$ was $4.39,69.90$, and 65.80 for $h \mathrm{AChE}$, and 133.07, 116.11, and 41.33 for $h \mathrm{BuChE}$. Autodock Tools were occupied with providing both grid and docking parameter files, i.e., gpf and dpf. Ligand-receptor interactions were all detected based on docking results using the Autodock tools program (ADT, Version1.5.6), VMD software [23], and PLIP (fully automated protein-ligand interaction profiler) [24]. This software calculates hydrogen bonding, arene-arene, arene-hydrogen, and hydrophobic interactions established through the interaction of ligands to their receptors.

\subsection{Model development.}

Two different regression methods were practical for QSAR equations, including simple multiple linear regression with stepwise variable selection (MLR), and Genetic algorithm-partial least squares (GA-PLS). These known methods are well-practiced in QSAR studies [25]. Here, stepwise selection and elimination of variables were practical for developing QSAR models using SPSS software (version21; SPSS Inc., IBM, Chicago, IL, USA). The leave-one-out crossvalidation procedure accredited the resulted models to test their predictability and robustness using MATLAB 2011 software (version 8.5; Math work Inc., Natick, MA, USA). The partial least square (PLS) regression method was applied to the NIPALS-based algorithm that existed within MATLAB software's chemometrics toolbox. The MATLAB PLS toolbox was used for PLS and GA modeling. All calculations were run on a Core i7 computer with the Windows 10 software.

\subsection{Model validation.}

Statistical parameters such as standard error of the regression (SE), correlation coefficient $\left(\mathrm{R}^{2}\right)$, variance ratio $(\mathrm{F})$ at specified degrees of freedom, leave-one-out crossvalidation correlation coefficient $\left(\mathrm{Q}^{2}\right)$, root mean square error of cross-validation (RMScv), and double cross-validation ( $\mathrm{Cvcv}$ ) were employed for the validity of regression equation [25]. To test the developed model efficiency, $20 \%$ of the molecules were selected as test set molecules. The predictive value of a QSAR model that was not considered during the development process has been evaluated on an external set of data. QSAR model was developed in more than three data sets, and the best equation was selected as the best model.

\subsection{Applicability domain.}

The uses of a QSAR model are predicated on its exact prediction ability for new compounds. Regardless of how considerable and accredit a QSAR could also be, it cannot be expected to reliably predict the modeled property for the whole space of chemicals. Before using QSAR for screening chemicals, its domain of application must be defined. Forecasts for chemicals during this area are reliable. The applicability domain is estimated by the leverage values for every compound. A Williams plot (the plot of standardized residuals versus leverage values (h)) can then be used for a moment and straightforward graphical detection of both the response outliers (Y outliers) and structurally influential chemicals (X outliers) in our model. During this graph, the applicability domain is appointed inside a squared area within $\pm \mathrm{X}$ (standard deviations) and a leverage threshold $\mathrm{h}^{*}$. 
The numerical value of leverage has certain characteristics: (a) the value is always greater than zero, (b) the lower the value; the upper is the confidence within the prediction. A worth of 1 indicates a very poor prediction. A price of 0 indicates perfect prediction and cannot be achieved. Another factor for analysis of the results is warning leverage $\left(\mathrm{h}^{*}\right)$. The threshold $\mathrm{h}^{*}$ is mostly fixed at $3(\mathrm{k}+1) / \mathrm{h}$ ( $\mathrm{k}$ is that the number of model parameters and $\mathrm{n}$ is that the number of coaching set compounds), whereas $x=2$ or 3. Prediction must be considered unreliable for compounds with a high leverage value $(h>h *)$. Leverage greater than warning leverage $\mathrm{h}^{*}$ means the expected response is that the results of substantial extrapolation of the model and thus might not be reliable. From the opposite point of view, when the leverage value of a compound is below the brink value, the probability of agreement between observed and predicted values is as high as that for the training set compounds [26, 27].

\subsection{MD simulation.}

Crystal structure of $h \mathrm{AChE}$ (PDB ID:1ACJ), $h \mathrm{BuChE}$ (4BDS) receptors, and the modified protein's model structure was used for theoretical studies. MD simulation and molecular mechanics (MM+) minimization were performed using Gromacs 5.0.7 package under an amber ff03force field. The GAFF topologies for ligands were generated using the Antechamber software using partial charges calculated with the restrained electrostatic potential method. The GAFF topologies were converted into GROMACS format using the acpype tool. MD simulations were applied with periodic boundary conditions. Van der Waals forces were treated with a cut-off of $10 \AA$. The Particle-Mesh Ewald method was used with a ten $\AA$ cut-off. The frequency to update the neighbor list was 5. The protonation state of gromacs package was accustomed to calculate the full charge of compounds. Within the simulation's beginning, the entire system was minimized using the steepest descent followed by conjugate gradients algorithms. Within the second step or equilibration step, heavy atoms were restrained employing a force constant of $1000 \mathrm{~kJ} \cdot \mathrm{mol}^{-1} \cdot \mathrm{nm}^{-2}$, and also, the solvent and ions were allowed to evolve. This was done through minimization and molecular dynamics within the NVT ensemble for $100 \mathrm{ps}$ and within the NPT ensemble for $100 \mathrm{ps}$. Then to get equilibrium geometry at $310 \mathrm{~K}$ and $1 \mathrm{~atm}$, the system's temperature was increased, and therefore the velocities at each step were reassigned consistent with Maxwell-Boltzmann distribution at that temperature and equilibrated for $100 \mathrm{ps}$. Temperature coupling was set to $0.1 \mathrm{ps}$, and pressure coupling to $2 \mathrm{ps}$. The Berendsen algorithm was used for the thermostat and barostat during the equilibration step. All bonds were constrained via the LINCS algorithm. Within the final step or production phase, a 100 ns MD simulation was performed under an NPT ensemble. To retain temperature and pressure stable within the production step, Nosé-Hoover thermostat and Parrinello-Rahman barostat were used with removing position restraints. The temperature was at $310 \mathrm{~K}$ with a time step of two fs [28].

\section{Results and Discussion}

In this study, a detailed QSAR study was developed using a combination of chemical, electronic, and substituent constant to explore structural parameters affecting VilazodoneTacrin hybrids that have been designed to inhibit $h \mathrm{AchE}$ and $h \mathrm{BuChE}$. Two chemometrics methods (i.e., stepwise MLR and GA-PLS) were applied for making a relationship between the biological activity and molecular descriptors. 


\subsection{MLR modeling.}

At first, separate stepwise selection-based MLR analyses were done using various styles of descriptors, and then, using the pool of all calculated descriptors, an MLR equation was acquired. The results for $h \operatorname{AchE}(\operatorname{Eq~1,2)}$ and $h \mathrm{BuChE}(\mathrm{Eq} 3,4)$ are shown in Table 2.

The calculated statistical parameters including regression coefficient for calibration set $\left(\mathrm{R}^{2}\right)$, correlation coefficient $\left(\mathrm{R}^{2} \mathrm{p}\right)$ for predicted set, standard error (SE), specified degrees of freedom $(\mathrm{F})$, the regression coefficient for leave one out cross-validation $\left(\mathrm{Q}^{2}\right), \mathrm{Cvcv}$, and RMScv which were utilized for validating the goodness of fit of the resulted QSAR equations are demonstrated in Table 2. GA-PLS was selected as the best equation in both tables.

Table 2. The results of different QSAR models with different types of dependent variables for $h$ AchE and

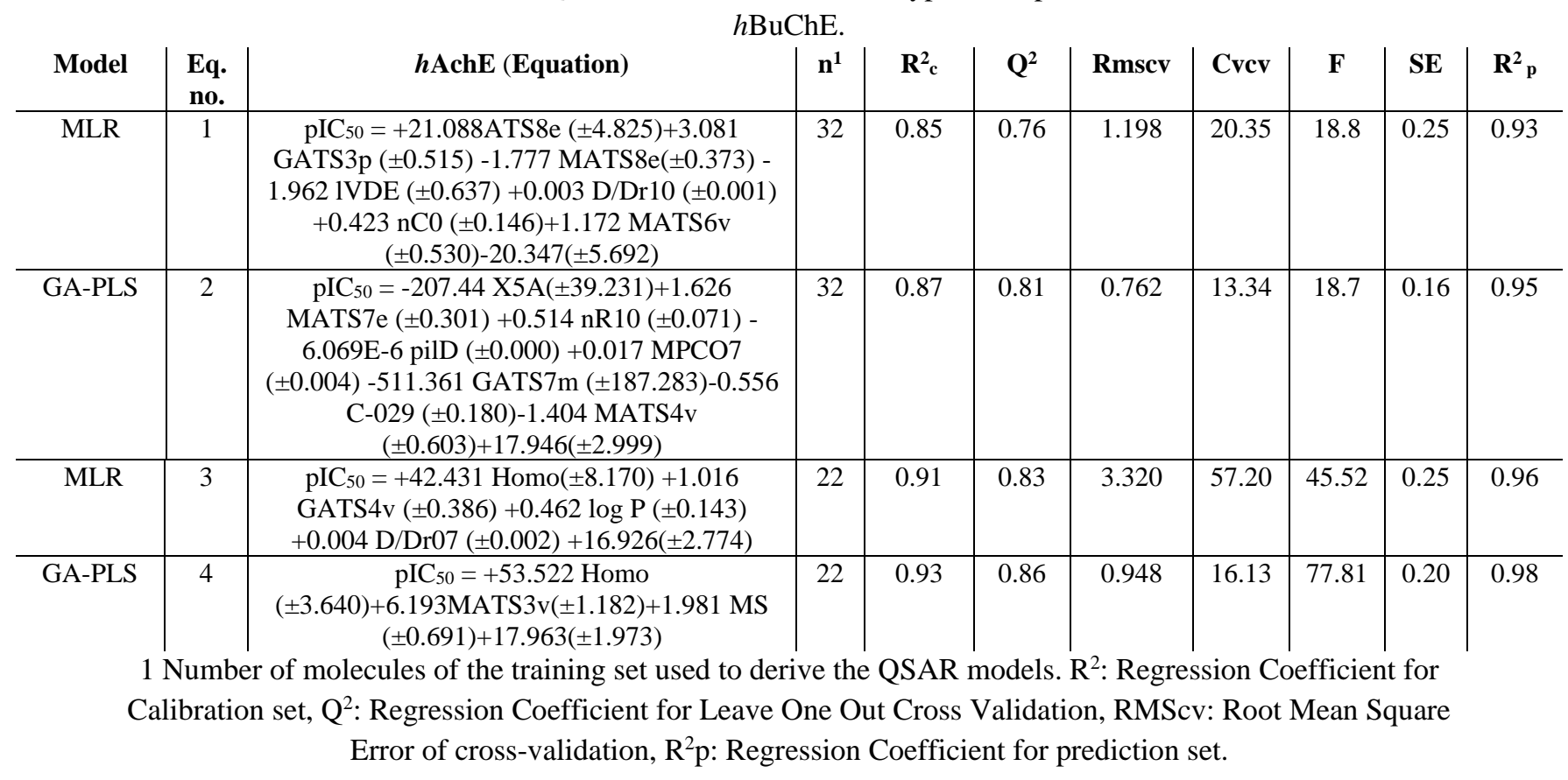

\subsection{GA-PLS.}

In PLS analysis, the descriptor's data matrix is decomposed into orthogonal matrices with an internal relationship between the dependent and independent variables. Hence, unlike MLR analysis, the multicollinearity problem within the descriptors is omitted by PLS analysis. Because PLS uses a small number of latent variables for modeling, it correlates with noisy data better than MLR. The calculated statistical parameters for this model are represented in Table 2 for both $h \mathrm{AChE}$, and $h \mathrm{BuChE}$.

The PLS estimate of coefficients for the descriptors is given in Figure 2A. As observed, a mixture of chemical, topological, geometrical, quantum, 2D-autocorrelations, and functional descriptors has been selected by GA-PLS to account for the hAChEI activity VilazodoneTacrine hybrids. The VIP analysis of the PLS equation is shown in Figure 2B.

VIP shows ATS8e, Softness, C025, IVDE, MATS8m, X4A, ATS3e, GATS8e, pipC05, $\mathrm{X} 3 \mathrm{~A}, \mathrm{VRA} 1$ and PCR are the most important index in the QSAR equation derived by PLS analysis. 


\subsection{In silico screening.}

In silico screening, studies are to replace time-consuming and costly in vivo experiments within the early stages of drug development.
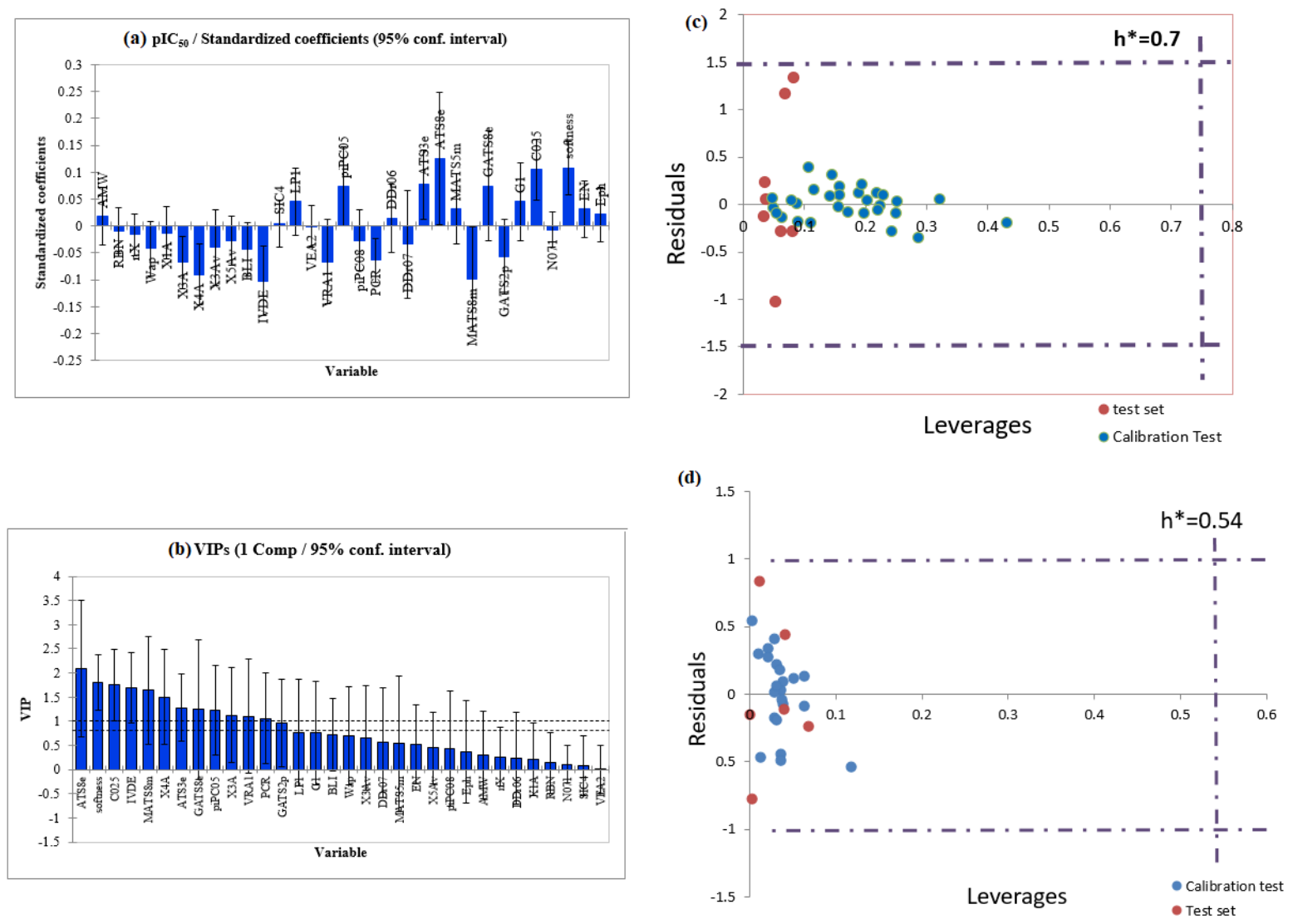

Figure 2. (a) PLS regression coefficients for the variables used in the GA-PLS model for hAChE; (b) Variable importance in the projection (VIP) for the variables used in the GA-PLS model for $h$ AChE. Williams plot for the calibration set and external prediction set for (c) $h \mathrm{AChE}$; (d) $h \mathrm{BuChE}$ of studied compounds.

It has the potential to hurry speed the rate of discovery, predicting, and identifying new pharmaceutical compounds. Virtual screening could be a potent technique to identify active compounds from molecular databases. It was applied by elimination, interpolation, and substitution of various substitutes on the parent molecules, and the effects of the structural modifications on the biological activity were investigated. The domain application of the QSAR model was resolute to use the model for screening new compounds. The applicability domain of the QSAR model was applied to verify the prediction reliability, acknowledge the strict compounds, and predict the compounds with the passable activity within this domain. We employed the important descriptors selected in both the GA-PLS model for designing new active compounds because of their greatest statistical parameters compared to the others. Analyzing the model applicability domain in the Williams plot of the GA-PLS model of $h \mathrm{AChE}$ (Figure 1C) and $h \mathrm{BuChE}$ (Figure 1D) supported the entire data set appeared that none of the compounds were identified as a clear outlier for this activity.

None of the compounds have leverage (h) values greater than the threshold leverages $\left(h^{*}\right)$. The warning leverage $\left(\mathrm{h}^{*}\right)$, was found to be 0.75 for $h \mathrm{AChE}$ and $0.54 \mathrm{for} h \mathrm{BuChE}$. The compounds that had a standardized remaining quite 3 times the standard deviation units were considered to be outliers. For both the training set and prediction set, the presented model matches the high-quality parameters with good fitting power and the ability to assess external data. Moreover, almost all of the compounds were within the proposed model's applicability 
domain and evaluated identically. Simultaneously, chemicals with a leverage value higher than $\mathrm{h}^{*}$ were considered influential or high leverage chemicals [29].

Based on the QSAR model, the in-silico screening was used to design new compounds with improved potential $h \mathrm{AChE} / h \mathrm{BuChE}$ inhibitor activity. As shown in Table 3, thirty novel compounds were designed and their predicted activities for both $h \mathrm{AChE}$ and $h \mathrm{BuChE}$ inhibitory activity based on GA-PLS equations and their docking binding energies were obtained. Leverage values show that all of the designed compounds were within the applicability domain. Among these molecules (Table 3), compounds 1, 6, 7, 8, 13, 15, 16s, 17s, $18 \mathrm{~s}, 20 \mathrm{~s}$, and $25 \mathrm{~s}$ showed the best activity. These compounds have good potential for becoming anti-Alzheimer agents.

Table 3. Structural modification of studied compounds and their predicted activities and docking binding energies for hAChE and hBuChE inhibitory based on GA-PLS equation.

\begin{tabular}{|c|c|c|c|c|c|c|c|}
\hline \multirow[b]{2}{*}{ Name } & \multirow[b]{2}{*}{ Compounds Structure } & \multicolumn{3}{|c|}{$h \mathrm{AChE}$} & \multicolumn{3}{|c|}{$h \mathrm{BuChE}$} \\
\hline & & pIC50 pred & $\begin{array}{c}\text { lever } \\
\text { age }\end{array}$ & $\begin{array}{c}\Delta \mathbf{E} \\
\text { (kcal/m } \\
\text { ol) } \\
\end{array}$ & $\begin{array}{l}\text { pIC }_{50} \\
\text { pred }\end{array}$ & $\begin{array}{c}\text { levera } \\
\text { ge }\end{array}$ & $\begin{array}{c}\Delta \mathbf{E} \\
(\mathrm{kcal} / \mathrm{m} \\
\text { ol) }\end{array}$ \\
\hline 1 & & 6.302 & 0.090 & -16.69 & 4.735 & 0.081 & -13.16 \\
\hline 2 & & 5.850 & 0.000 & -14.90 & 4.712 & 0.084 & -11.99 \\
\hline 3 & & 6.076 & 0.024 & -15.25 & 5.204 & 0.026 & -12.97 \\
\hline 4 & & 6.076 & 0.024 & -15.22 & 5.384 & 0.012 & -13.28 \\
\hline 5 & & 6.076 & 0.024 & -16.01 & 4.862 & 0.063 & -13.27 \\
\hline 6 & & 6.302 & 0.090 & -14.60 & 5.144 & 0.031 & -13.32 \\
\hline 7 & & 6.528 & 0.183 & -14.07 & 5.351 & 0.014 & -12.51 \\
\hline 8 & & 6.302 & 0.090 & -13.03 & 6.092 & 0.007 & -12.33 \\
\hline
\end{tabular}




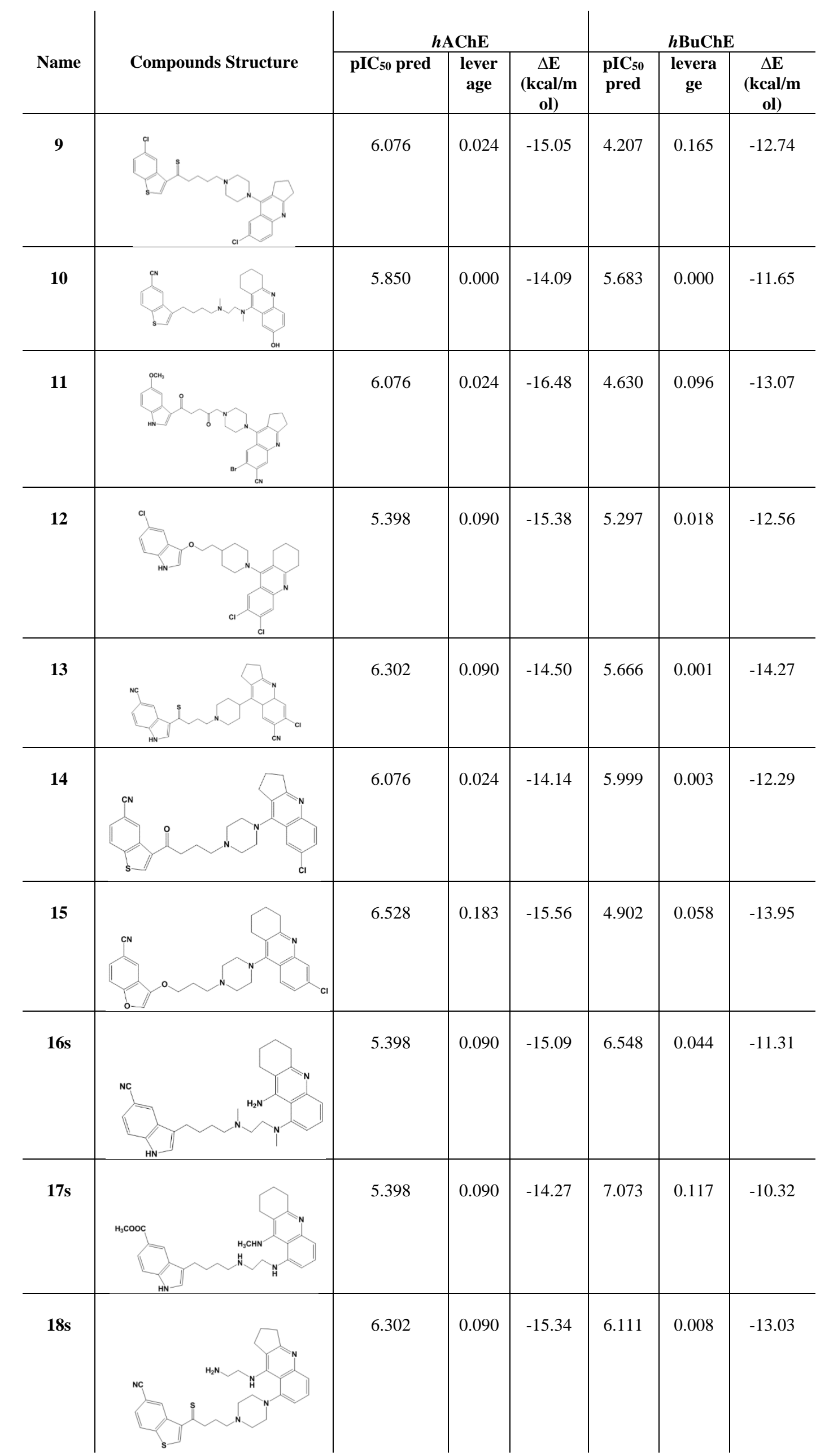




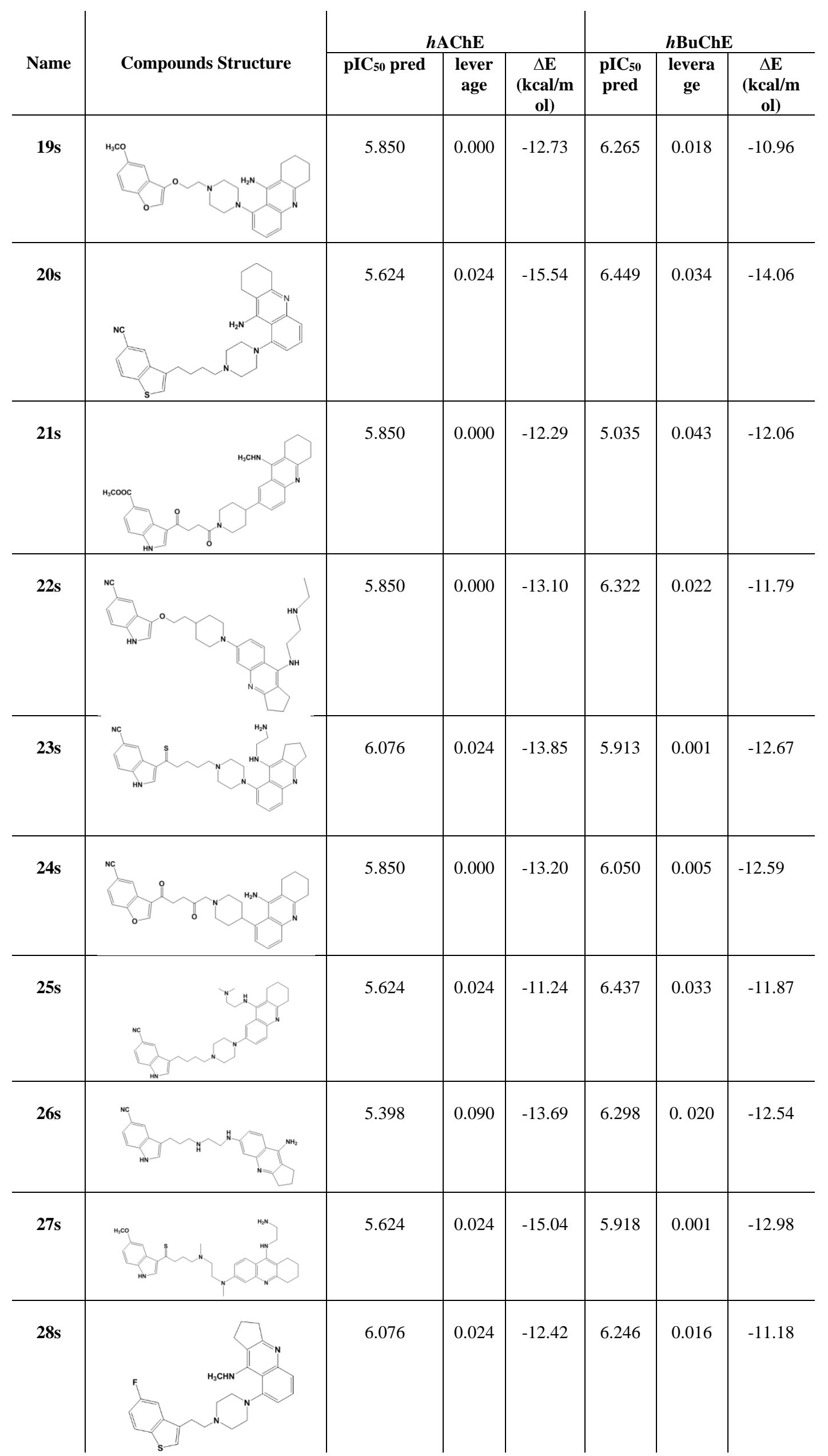




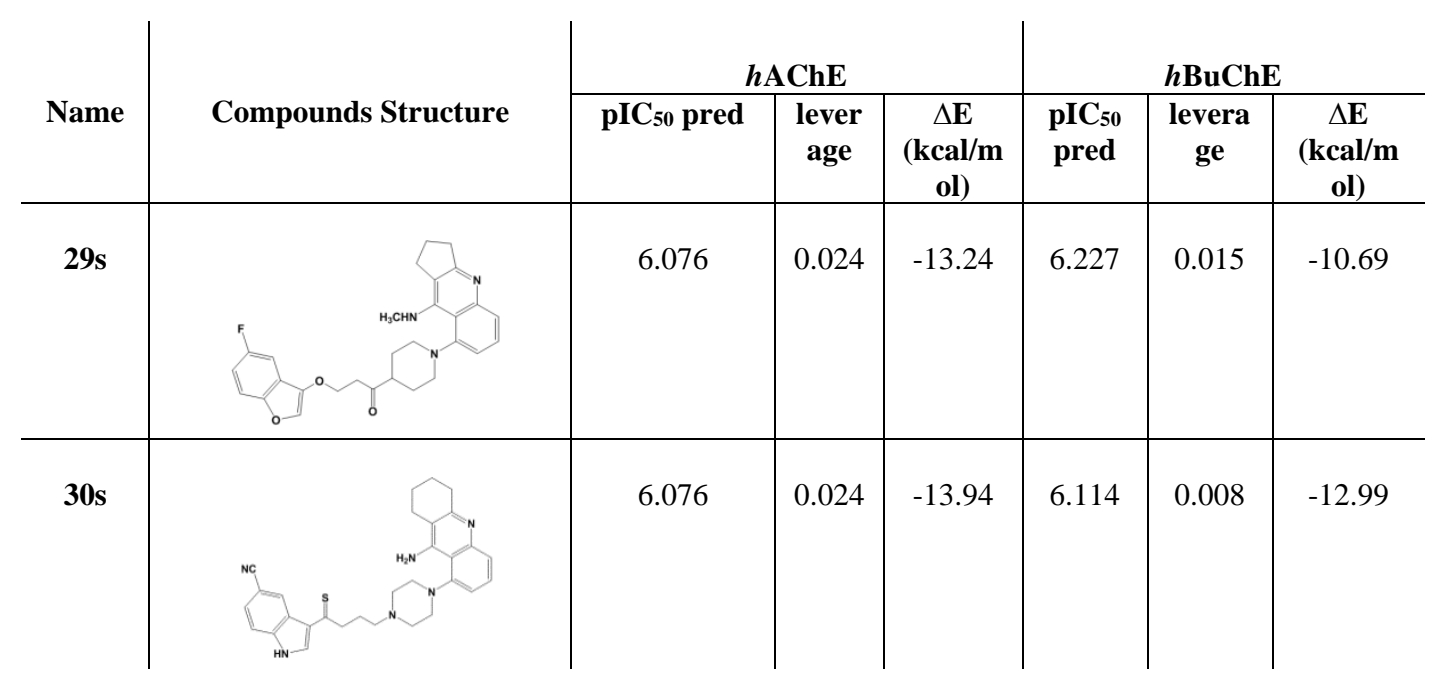

To have attention on the cross-validated prediction results, the predicted activity data are plotted against the experimental activities in Figure 3. The least scattering of data was obtained from GA-PLS for $h \mathrm{AChE}$ and $h \mathrm{BuChE}$, respectively.
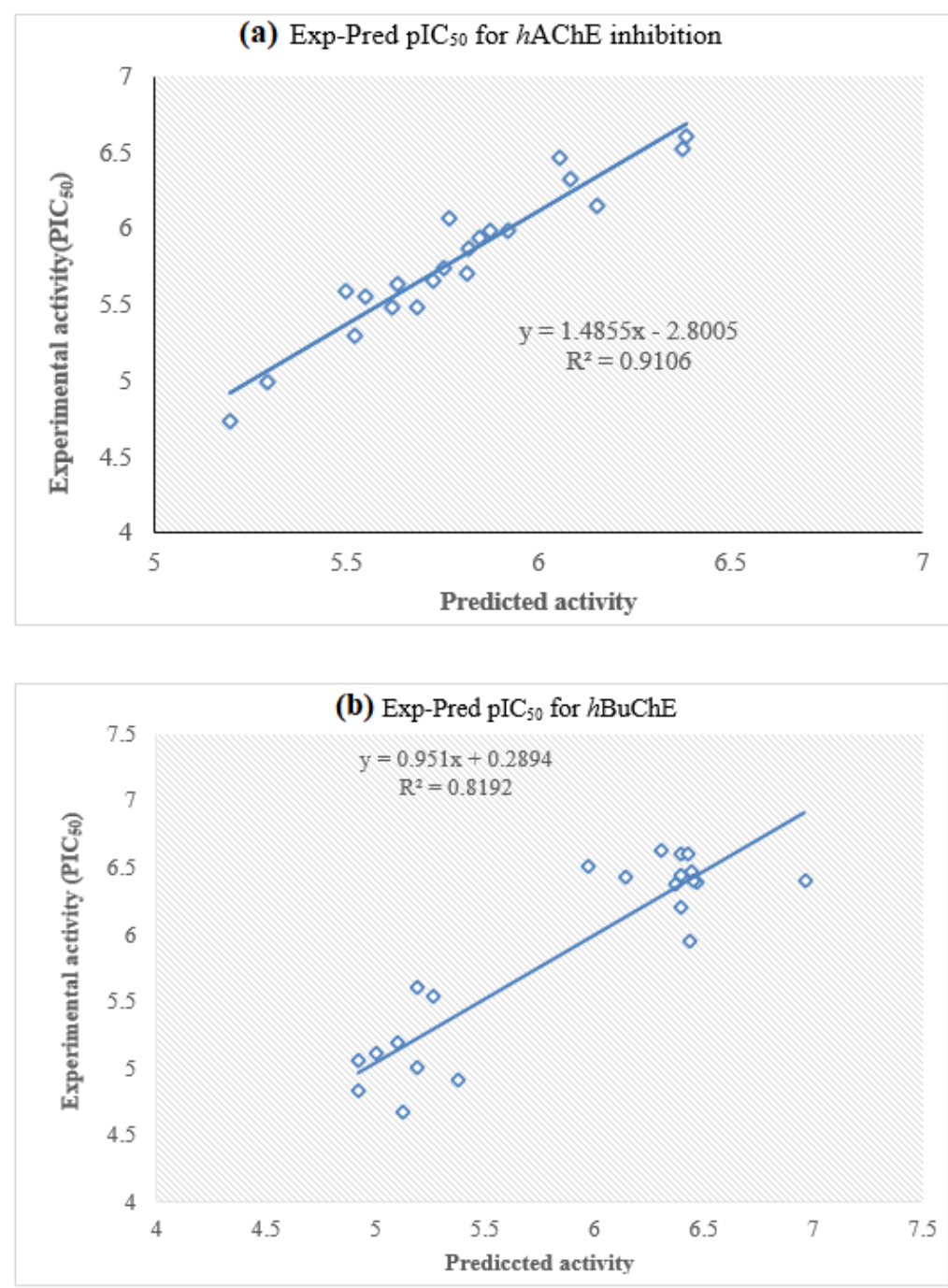

Figure 3. (a) Plots of cross-validated predicted values of activity by GA-PLS against the experimental values ( $h$ AChE). (b) Plots of cross-validated predicted values of activity by GA-PLS against the experimental values ( $h \mathrm{BuChE})$. 
The high regression ratio $\left(\mathrm{R}^{2}=0.91,0.82\right.$ for $h \mathrm{AChE}$ and $\left.h \mathrm{BuChE}\right)$ in this plot shows the great agreement between cross-validated predicted values of activity and the experimental activity.

\subsection{Molecular Docking Studies}

All of the dataset compounds as well as the designed compounds were subjected to molecular docking studies on $h \mathrm{AChE}$ (PDB ID:1ACJ), and $h \mathrm{BuChE}$ (4BDS) receptors. The estimated free binding energy values ( $\Delta$ Gbind) in $\mathrm{kcal} \mathrm{mol}^{-1}$ and the interactions with the key amino acid residues in the active site of enzymes were completely determined (Table 1, 3, and Figures 5, 6).

Redocking of tacrine as the co-crystal ligand of $h \mathrm{AChE}$, and $h \mathrm{BuChE}$ with a high affinity for these targets indicated that the X-ray crystallography conformer was extremely identical to the docked conformer. RMSD of docking for tacrine compared to its coordination in the crystal structure was 0.67 and 0.63 for $h \mathrm{AChE}$, and $h \mathrm{BuChE}$, respectively (Figure 4).

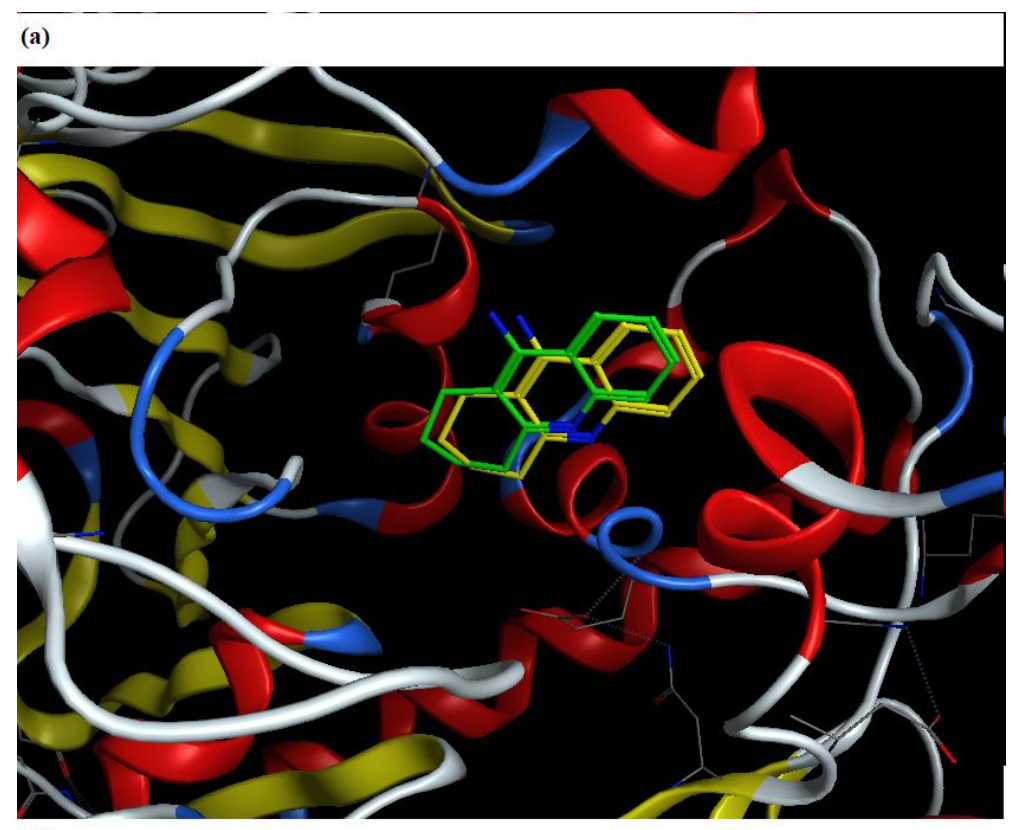

(b)

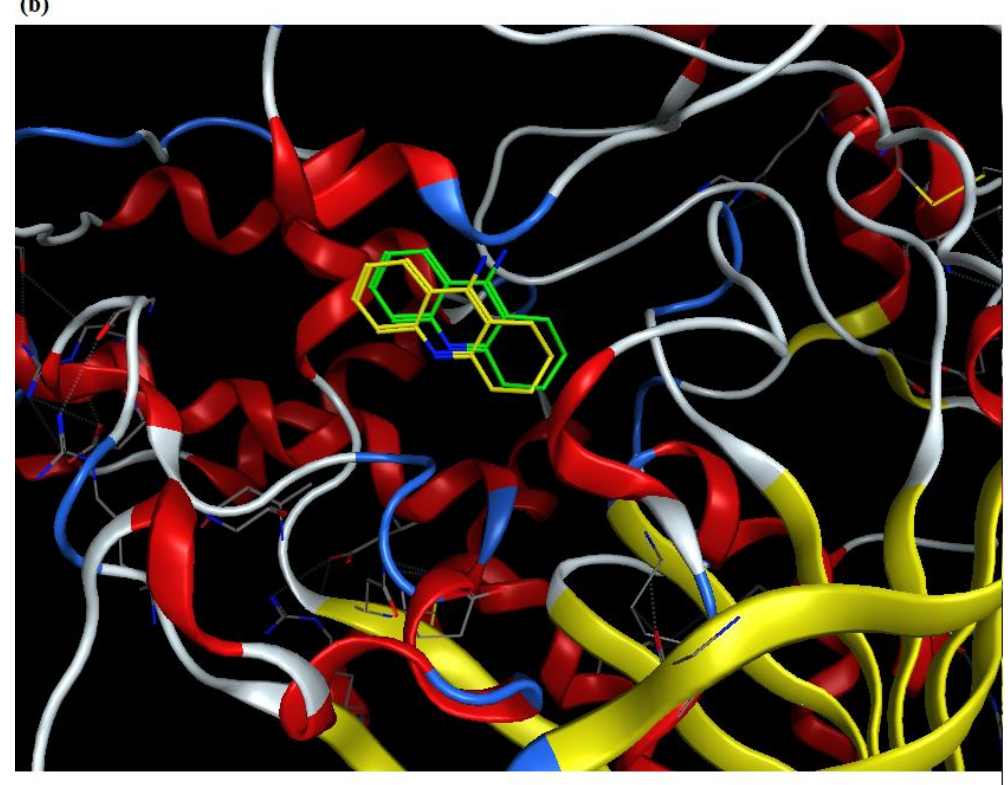

Figure 4. Comparison of two bound conformations of tacrine in the active site of (a) $h \mathrm{AChE}$, and (b) $h \mathrm{BuChE}$ : the yellow model shows the crystal orientation, and the redocked result is shown in green. 
As it was shown in Table 1, the dataset can classify into two different classes. The structural changes in these compounds have affected the docking results. The $\Delta$ Gbind values of the best-docked poses of series A (compounds A1-A23) are within the range of -11.54 to $16.45 \mathrm{kcal}_{\mathrm{mol}}^{-1}$ for $h \mathrm{AChE}$ and -6.51 to $-16.53 \mathrm{kcal}^{-\mathrm{mol}^{-1}}$ for $h \mathrm{BuChE}$. The best docking binding energies of series B (compounds B1-B16) are within the range of -10.38 to -13.31 kcal.mol ${ }^{-1}$ for $h \mathrm{AChE}$ and -10.25 to $-14.19 \mathrm{kcal} . \mathrm{mol}-1$ for $h \mathrm{BuChE}$. The docking binding energy of tacrine (the co-crystal ligand of both receptors) is -8.71 and $-7.55 \mathrm{kcal} . \mathrm{mol}-1$ on hAChE $h \mathrm{BuChE}$, respectively.
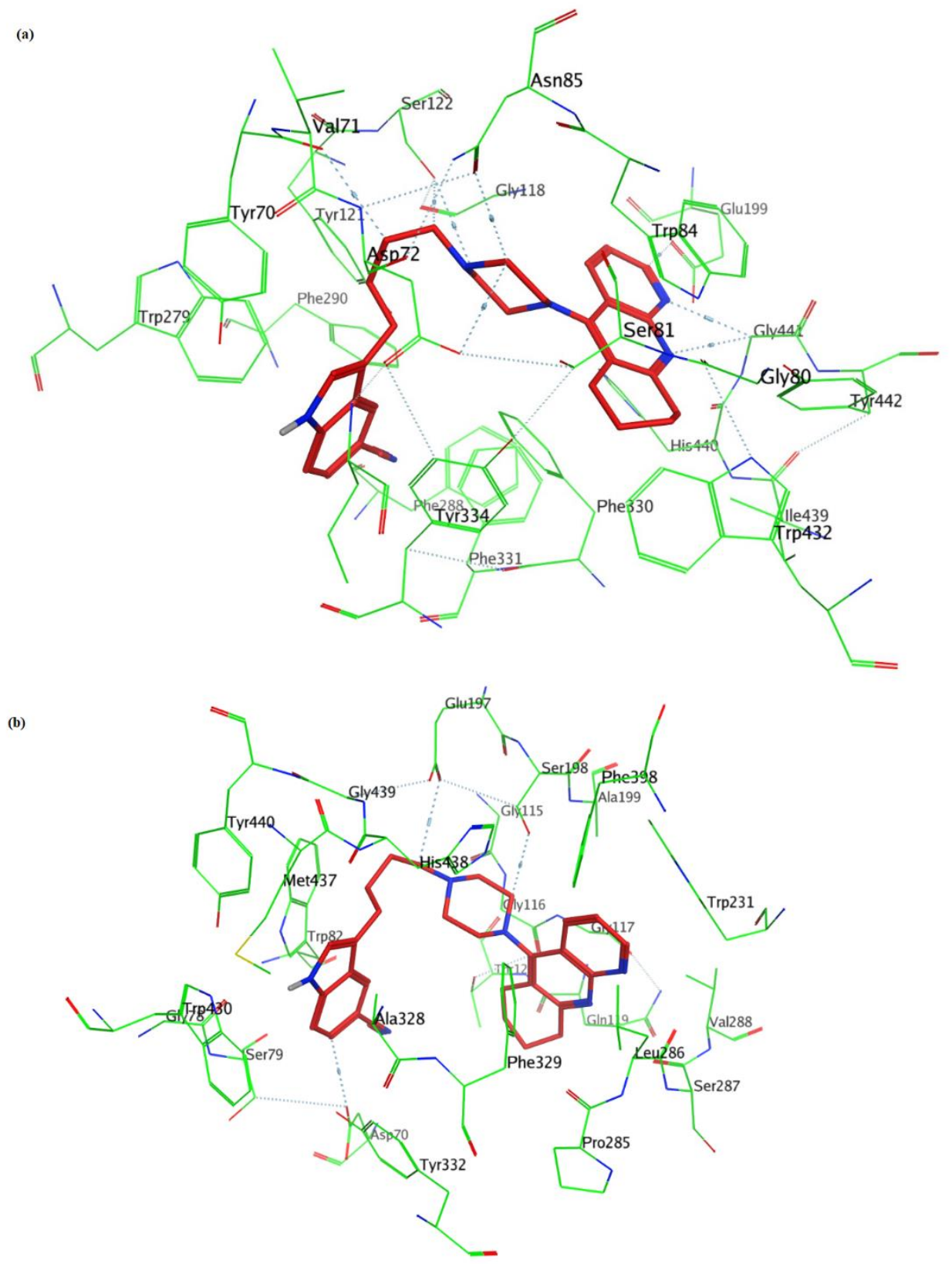

Figure 5. The structure of A 2 surrounded by the key residues in the active site of (a) $h \mathrm{AChE}$; (b) $h \mathrm{BuChE}$.

As indicated in Figure 5a, in the $h \mathrm{AChE}$ binding mode of A2, the nitrogen of pyridine and piperazine rings are involved in hydrogen bond interactions with residues Gly441 and Ser122, respectively. The pyridine rings of tacrine moiety are involved in $\pi$ - $\pi$ interactions with residues Trp84. The side chains consisting of amino acid residues Glu199, Asp72, and Asp85 make direct van der Waals contacts with compound A2. 
The binding mode of $\mathrm{A} 2$ with $h \mathrm{BuChE}$ (figure $5 \mathrm{~b}$ ) shows that the $\mathrm{NH}$ of the indole ring is involved in arene-hydrogen interactions with residues Trp430. A $\pi-\pi$ interaction between phenyl of indole ring and residues Trp82 is also visible. There is also existed acceptor hydrogen bond interaction between the nitrogen of piperazine ring with residues Glu197. Some hydrophobic interactions with residue Asp70 and Ser196 also exist.

Molecular docking was also applied to the designed compounds to gain some more insight into their binding mode to the $h \mathrm{AChE}$ and $h \mathrm{BuChE}$ targets. The binding mode of $18 \mathrm{~s}$ in the active site of $h \mathrm{AChE}$ and $h \mathrm{BuChE}$ is evaluated in Figures 6.

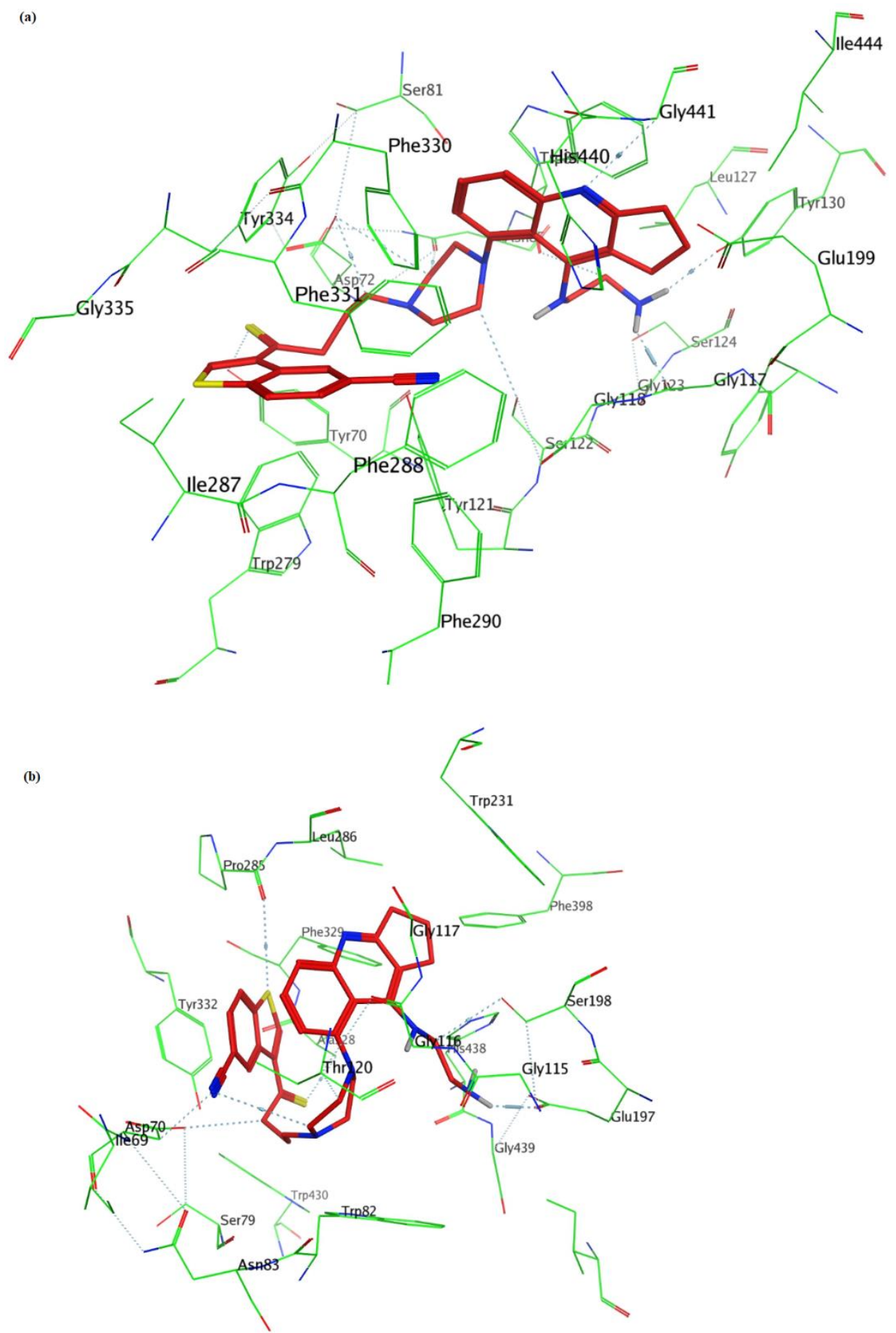

Figure 6. The structure of 18 s surrounded by the key residues in the active site of (a) $h \mathrm{AChE}$; (b) $h \mathrm{BuChE}$.

As it was depicted in figure 6a, the key amino acids in the binding mode of compound $18 \mathrm{~s}$ with $h \mathrm{AChE}$ are two arene-arene interactions between phenyl and pyridine rings with Trp84. Three hydrogen bonding exists between the nitrogen atom of the pyridine ring, $\mathrm{NH}$ of https://biointerfaceresearch.com/ 
piperazine, and NH of amino side chain on tacrine with Gly441, Asp72, and Tyr130, respectively. Some hydrophobic interactions with residue Asp72, Asp85, and Trp84 are also observed. In the binding of $18 \mathrm{~s}$ to $h \mathrm{BuChE}$ (Figure 6b), the NH of amino side chain on tacrine, sulfur of thiophene ring, and sulfur of $\mathrm{C}=\mathrm{S}$ group are involved in hydrogen bonding with Glu197, Pro285, and Ala328, respectively. There is also existed some hydrophobic interactions with residue Asp70, and Glu197. The thiophene ring is also involved in $\pi-\pi$ interaction with Tyr332. Some hydrophobic interactions with residue Ser198, Asp70, and Thr120 also exist.

The results obtained from this docking study indicate that the important amino acids inside the active site cavity that are in charge of essential interactions are Gly441, Ser122, Trp84, Asp72, Tyr130, and Gly117 for $h$ AChE and Glu197, Thr120, Gly121, Asn83, Tyr332, Trp430, Trp82 and Asp70 for $h \mathrm{BuChE}$. The majority of the studied compounds took part in hydrogen bond formation especially through the hydrogen attached to nitrogen of pyridine with Gly441 for $h \mathrm{AChE}$, and nitrogen of piperazine with Glu197 for $h \mathrm{BuChE}$, respectively. It should be noted that high activity compounds form one or more hydrogen bonds with the active site residues. Therefore, one of the essential requirements for optimum $h \mathrm{AChE}$ and $h \mathrm{BuChE}$ inhibition is hydrogen bond formation by ligands. The docking results suggest that apart from hydrogen bonding formation, the binding of different compounds with the active site is stabilized by van der Waals and hydrophobic interactions with the non-polar amino acids. The arene-arene interaction also exists between ligands and their targets through their phenyl, pyridine, thiophene, and pyrrole rings.

\subsection{MD simulation.}

Root Mean Square Deviation (RMSD) deviation between the created structures within the simulation over time is an appropriate and common criterion to ensure the stability of molecular dynamics simulations. Therefore, the RMSD changes of protein alpha carbon atoms during the simulation time (100 ns) were calculated and extracted from the initial structure. The results of this calculation are given in Figure 6a.

As shown in Figure 7a, the protein RMSD diagram shows the steep gradient with the RMSD reaching $0.14 \mathrm{~nm}$ up to $5000 \mathrm{ps}$ of molecular dynamics simulation. From 5000 to 95,000 ps the incremental slope has been slowed. At 95,000 ps, the rate of RMSD changes increased by $0.2 \mathrm{~nm}$. Also, as shown in Figure 7a, the RMSD increased with a steep slope after $95000 \mathrm{ps}$. So that at the end of the molecular dynamics simulation (100,000 ps), the RMSD is equal to $0.26 \mathrm{~nm}$. This indicates a sudden change in the structure of part of the protein. Next, to investigate the ligand stability in the protein-bound state, the RMSD of this molecule was also studied (Figure 7b). As shown in Figure 7b, the ligand molecule is rapidly stabilized at the beginning of the simulation time, but after 60,000 ps of the diagram simulation, there is a strong oscillation indicating a slight displacement of the ligand in the binding site.

\subsubsection{Molecular flexibility examination.}

The dynamic behavior of alpha carbon atoms in the protein structure contains sufficient information to investigate important motions in proteins and reflects the general structure motions [30]. Therefore, the root means square fluctuation (RMSF) of the alpha carbon atoms was considered to investigate the structural mobility and flexibility (Figure 7c). The RMSF diagram for protein in the range of 80 to $100 \mathrm{~ns}$ in alpha carbon is shown in Figure 7c. As can be seen, the carboxyl end amino acids exhibit high flexibility. This graph's data are consistent 
with the increase in RMSD (Figure 7a) after 80,000 picoseconds of molecular dynamics simulation.

\subsubsection{Radius of gyration.}

The radius of gyration as an important factor indicates the protein's stability in the simulation environment [30]. So, suppose the protein is well stable in the simulation medium. In that case, the average radius for that protein during the simulation period is constant. The decrease in gyration radius during the simulation time indicates that the protein is compressed, and the increase in radius showed the opposite result. The variation of the gyration radius during the simulation time (100 ns) for the protein molecule is shown in Figure $7 \mathrm{~d}$.

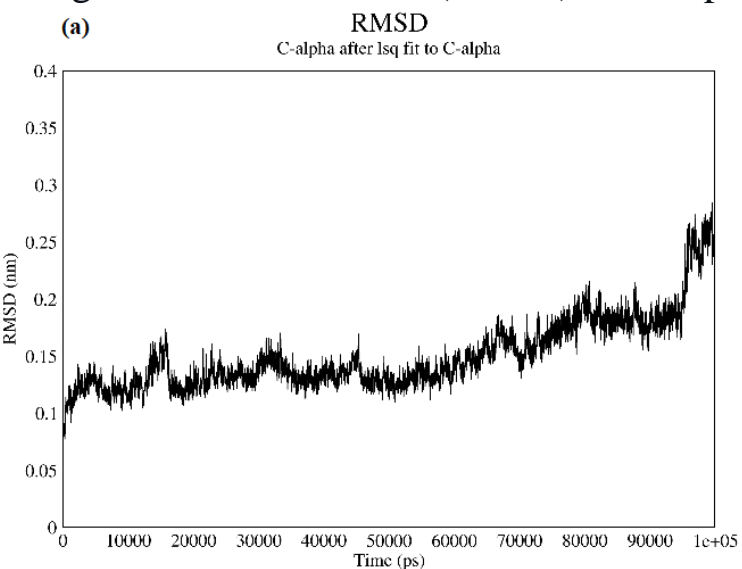

(c) RMS fluctuation

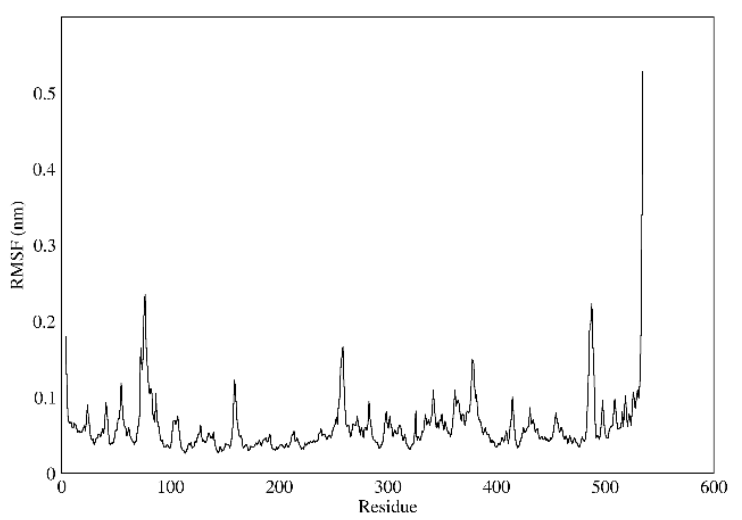

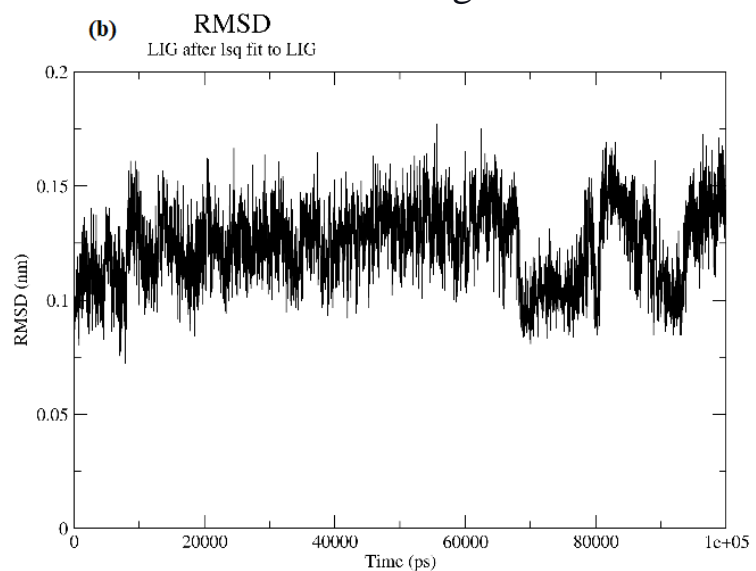

(d) Radius of gyration (total and around axes)

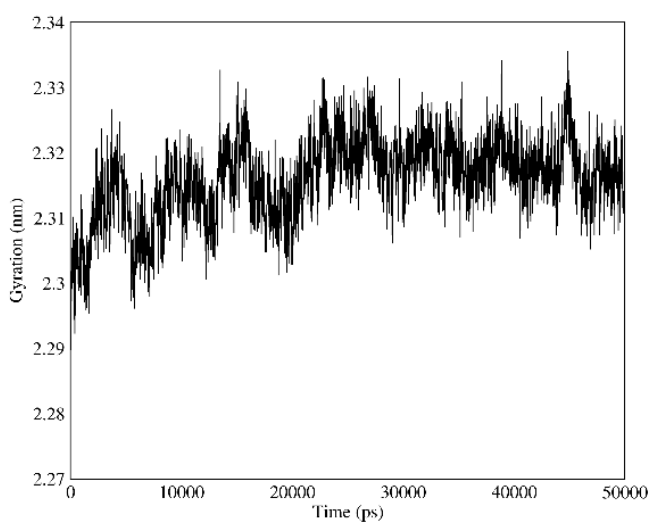

Figure 6. MD simulation results. (a) The RMSD changes of protein alpha carbon atoms during the simulation time (100 ns); (b) The RMSD of the ligand 18s; (c) The root means square fluctuation (RMSF) of the alpha carbon atoms; (d) The variation of the gyration radius during the simulation time (100 ns) for the protein molecule.

\section{Conclusions}

Quantitative relationships between molecular structure and $h \mathrm{AChE}$ and $h \mathrm{BuChE}$ activity of a series Vilazodone-Tacrine hybrids were discovered by chemometrics methods including MLR and GA-PLS. The reliability, accuracy, and predictability of the proposed models were evaluated. The docking study indicates that the important amino acids inside the active site cavity in charge of essential interactions were Gly441, Ser122, Trp84, Asp72, Tyr130 Gly117 for $h$ AChE and Glu197, Thr120, Gly121, Asn83, Tyr332, Trp430, Trp82, and Asp70 for $h \mathrm{BuChE}$. Furthermore, these compounds showed proper interactions, including ionic bond, H-bond, and $\pi-\pi$ interactions. The best regression equation was obtained from GAPLS, which predicts $92 \%$ of variances. Finally, an in silico screening study was conducted, and 
some compounds were designed as inhibitors of $h \mathrm{AChE}$ and $h \mathrm{BuChE}$. Among them, compounds $1,6,7,8,13,15,16 \mathrm{~s}, 17 \mathrm{~s}, 18 \mathrm{~s}, 20 \mathrm{~s}$, and $25 \mathrm{~s}$ showed the best activity. These compounds have good potential for becoming anti-Alzheimer agents.

\section{Funding}

This research was funded by Ahvaz Jundishapur University of Medical Sciences, grant number U-98106.

\section{Acknowledgments}

The authors would like to thank research deputy Ahvaz Jundishapur University of Medical Sciences, who supports this work. The collaboration of Medicinal Chemistry, Faculty of Pharmacy, Ahvaz Jundishapur University of Medical Sciences, in providing the required facilities for this work is greatly acknowledged. This article is extracted from the project with grant Number: U-98106 and Ethic: IR.AJUMS.REC.1398.436).

\section{Conflicts of Interest}

The authors declare no conflict of interest

\section{References}

1. Gaugler, J.; James, B.; Johnson, T.; Marin, A.; Weuve, J. 2019 Alzheimer's disease facts and figures. Alzheimers \& Dementia 2019, 15, 321-387, https://doi.org/10.1016/j.jalz.2019.01.010.

2. Raza, M.; Awais, M.; Ellahi, W.; Aslam, N.; Nguyen, H.X.; Le-Minh, H. Diagnosis and Monitoring of Alzheimer's Patients Using Classical and Deep Learning Techniques. Expert Syst. Appl. 2019, 136, 353-364, https://doi.org/10.1016/j.eswa.2019.06.038.

3. Pan, H.; Zhang, J.; Wang, Y.; Cui, K.; Cao, Y.; Wang, L.; Wu, Y. Linarin Linarin improves the dyskinesia recovery in Alzheimer's disease zebrafish by inhibiting the acetylcholinesterase activity. Life. sci. 2019, 222, 112-116, https://doi.org/10.1016/j.lfs.2019.02.046.

4. Ulep, M.G.; Saraon, S.K.; McLea, S. Alzheimer disease. The Journal for Nurse Practitioners 2018, 14, 129 135, https://doi.org/10.1016/j.nurpra.2017.10.014.

5. Vilela, A.F.L.; Cardoso, C.L.; Mateo, C. An immobilized acetylcholinesterase as test system to screen new inhibitor drugs to treat Alzheimer's disease. Sens. Actuators B. Chem. 2019, 278, 196-201. https://doi.org/10.1016/j.snb.2018.09.071.

6. Liu, W.; Wang, H.; Li, X.; Xu, Y.; Zhang, J.; Wang, W.; Gong, Q.; Qiu, X.; Zhu, J.; Mao, F.; Zhang, H. Design, synthesis and evaluation of vilazodone-tacrine hybrids as multitarget-directed ligands against depression with cognitive impairment. Bioorg. med. chem. 2018, 26, 3117-3125, https://doi.org/10.1016/j.bmc.2018.04.037.

7. Manouchehrizadeh, E.; Mostoufi, A.; Tahanpesar, E.; Fereidoonnezhad, M. Alignment-Independent 3DQSAR and Molecular Docking Studies of Tacrine-4-oxo-4H-Chromene Hybrids as anti-Alzheimer's Agents. Comput. Biol. Chem. 2019, 80, 463-471, https://doi.org/10.1016/j.compbiolchem.2019.05.010.

8. Safarizadeh, H.; Garkani-Nejad, Z. Molecular docking, molecular dynamics simulations and QSAR studies on some of 2-arylethenylquinoline derivatives for inhibition of Alzheimer's amyloid-beta aggregation: Insight into mechanism of interactions and parameters for design of new inhibitors. J. Mol. Graph. Model. 2019, 87, 129-143, https://doi.org/10.1016/j.jmgm.2018.11.019.

9. Ultra, C., 12.0, Cambridge Soft, Cambridge, MA 2010. Received: May, 2013.

10. Frisch, MJ.; Gaussian 09 (Gaussian, Inc., Wallingford, CT), Revision D. 01. Becke AD (1988) Densityfunctional exchange-energy approximation with correct asymptotic behavior. Phys. Rev. A. Gen. Phys. 2009, 38, 3098-100, https://doi.org/10.1103/physreva.38.3098.

11. Mauri, A.; Consonni, V.; Pavan, M.; Todeschini, R. Dragon software: An easy approach to molecular descriptor calculations. Match. 2006, 56, 237-248.

12. Thanikaivelan, P.; Subramanian, V.; Rao, JR.; Nair, BU. Application of quantum chemical descriptor in quantitative structure activity and structure property relationship. Chem. Phys. Lett., 2000, 323, 59-70, https://doi.org/10.1016/S0009-2614(00)00488-7. 
13. Liu, X.; Yang, X.; Guo, R. Regional differences in fossil energy-related carbon emissions in China's eight economic regions: Based on the Theil index and PLS-VIP method. Sustainability 2020, 12, 2576, https://doi.org/10.3390/su12072576.

14. Chen, X.; Huang, J.; Yi, M. Cost estimation for general aviation aircrafts using regression models and variable importance in projection analysis. Journal of Cleaner Production 2020, 256, 120648, https://doi.org/10.1016/j.jclepro.2020.120648.

15. Fereidoonnezhad, M.; Faghih, Z.; Mojaddami, A.; Tabaei, SM.; Rezaei, Z. Novel approach synthesis, molecular docking and cytotoxic activity evaluation of N-phenyl-2, 2-dichloroacetamide derivatives as anticancer agents. J. Sci. I. R. Iran. 2016. 27, 39-49, https://doi.org/10.22059/JSCIENCES.2016.56007.

16. Fereidoonnezhad, M.; Faghih, Z.; Mojaddami, A.; Sakhteman, A.; Rezaei, Z. A comparative docking studies of dichloroacetate analogues on four isozymes of pyruvate dehydrogenase kinase in humans. Indian. J. Pharm. Edu. Res. 2016, 50, S32-S38.

17. G Keivanloo, A.; Abbaspour, S.; Sepehri, S.; Bakherad, M. Synthesis, Antibacterial Activity and Molecular Docking Study of a Series of 1, 3-Oxazole-Quinoxaline Amine Hybrids. Polycyclic Aromatic Compounds 2020, 1-14, https://doi.org/10.1080/10406638.2020.1833052.

18. Morris, G.M.; Huey R.; Olson, A.J. Using autodock for ligand-receptor docking. Curr. Protoc. Bioinformatics. 2008. 24, 8-14, https://doi.org/10.1002/0471250953.bi0814s24.

19. Hikisz, P.; Szczupak, Ł.; Koceva-Chyła, A.; Guśpiel, A.; Oehninger, L.; Ott, I.; Therrien, B.; Solecka, J.; Kowalski, K. Anticancer and antibacterial activity studies of gold (I)-alkynyl chromones. Molecules, 2015, 20, 19699-19718, https://doi.org/10.3390/molecules201119647.

20. Khan, A.; Al-Quaderi, G.D.; Bhuiyan, M.A.; Maria, K.H.; Choudhury, S.; Hossain, K.M.A.; Saha, D.K.; Akhter, S. Study of the magnetic properties of $\mathrm{Zn}$ doped Cobalt ferrite (CoZnxFe2-xO4). Biointerface Research in Applied Chemistry 2020, 10, 5665-5669, https://doi.org/10.33263/briac103.665669.

21. Hamedi, A.; Khoshnoud, MJ.; Tanideh, N.; Abbasi, F.; Fereidoonnezhad, M.; Mehrabani, D. Reproductive toxicity of Cassia absus seeds in female rats: possible progesteronic properties of chaksine and b-sitosterol. Pharm. Chem. J. 2015, 49, 268-274, https://doi.org/10.1007/s11094-015-1268-y.

22. Iftikhar, H.; Ali, H.N.; Farooq, S.; Naveed, H.; Shahzad-ul-Hussan, S. Identification of potential inhibitors of three key enzymes of SARS-CoV2 using computational approach. Computers in biology and medicine 2020, 122, 103848, https://doi.org/10.1016/j.compbiomed.2020.103848.

23. Wang, J.; Vanga, S.; K., \& Raghavan, V. (2020). Structural responses of kiwifruit allergen Act d 2 to thermal and electric field stresses based on molecular dynamics simulations and experiments. Food \& function 2020, 11, 1373-1384, https://doi.org/10.1039/C9FO02427A.

24. Salentin, S.; Schreiber, S.; Haupt, VJ.; Adasme, MF.; Schroeder, M. PLIP: fully automated protein-ligand interaction profiler. Nucleic Acids Res. 2015. 43, W443-W447, https://doi.org/10.1093/nar/gkv315.

25. Fereidoonnezhad M, Tabaei SM, Sakhteman A, Seradj H, Faghih Z, Faghih Z, Mojaddami A, Sadeghian B, Rezaei Z. Design, synthesis, molecular docking, biological evaluations and QSAR studies of novel dichloroacetate analogues as anticancer agent. J. Mol. Struct. 2020, 1221, 128689, https://doi.org/10.1016/j.molstruc.2020.128689.

26. Roy, K.; Kar, S.; Das, R.N. Understanding the basics of QSAR for applications in pharmaceutical sciences and risk assessment. Boston: Academic Press; 2015, 1-46.

27. Huang, Y.; Li, T.; Zheng, S.; Fan, L.; Su, L.; Zhao, Y.; Xie, HB.; Li, C. QSAR modeling for the ozonation of diverse organic compounds in water. Science of The Total Environment. 2020, 715, 136816, https://doi.org/10.1016/j.scitotenv.2020.136816.

28. Razzaghi-Asl, N. and A. Ebadi, In silico design of peptide inhibitors of tubulin: amyloid- $\beta$ as a lead compound. J. Biomol. Struct. Dyn. 2020, 1-10, https://doi.org/10.1080/07391102.2020.

29. Fereidoonnezhad, M.; Faghih, Z.; Mojaddami, A.; Rezaei, Z.; Sakhteman, A. A comparative QSAR analysis, molecular docking and PLIF studies of some N-arylphenyl-2,2-dichloroacetamide analogues as anticancer agents. Iran. J. Pharm. Res. 2017, 16, 981-998, https://doi.org/10.22037/IJPR.2017.2064.

30. Dastan, D.; Validi, S.; Ebadi, A. Kamonolol acetate from Ferula pseudalliacea as AChE inhibitor: in vitro and in silico studies. Struct. Chem. 2020, 31, 965-973, https://doi.org/10.1007/s11224-019-01473-z. 